\title{
André Pézard, autobiographe, italianiste, romaniste et médiéviste (1893-1984). Pour un profil intellectuel, dir. Michèle Gally et Elsa Marguin-Hamon, Paris, Classiques Garnier, 2017, 375 p.
}

\section{Monica Zanardo}

\section{OpenEdition}

\section{Journals}

Édition électronique

URL : https://journals.openedition.org/genesis/3234

DOI : 10.4000/genesis.3234

ISSN : 2268-1590

Éditeur :

Presses universitaires de Paris Sorbonne (PUPS), Société internationale de génétique artistique littéraire et scientifique (SIGALES)

\section{Édition imprimée}

Date de publication : 15 décembre 2018

Pagination : 175-177

ISBN : 979-10-231-06282

ISSN : 1167-5101

Référence électronique

Monica Zanardo, «André Pézard, autobiographe, italianiste, romaniste et médiéviste (1893-1984). Pour un profil intellectuel, dir. Michèle Gally et Elsa Marguin-Hamon, Paris, Classiques Garnier, 2017, 375 p. », Genesis [En ligne], 47 | 2018, mis en ligne le 15 janvier 2019, consulté le 10 octobre 2022. URL : http:// journals.openedition.org/genesis/3234; DOI : https://doi.org/10.4000/genesis.3234

Ce document a été généré automatiquement le 10 octobre 2022.

Tous droits réservés 


\section{André Pézard, autobiographe, italianiste, romaniste et médiéviste (1893-1984). Pour un profil intellectuel, dir. Michèle Gally et Elsa Marguin- Hamon, Paris, Classiques Garnier, 2017, 375 p.}

Monica Zanardo

\section{RÉFÉRENCE}

André Pézard, autobiographe, italianiste, romaniste et médiéviste (1893-1984). Pour un profil intellectuel, dir. Michèle Gally et Elsa Marguin-Hamon, Paris, Classiques Garnier, 2017,375 p.

Professeur de littérature italienne, spécialiste de Dante, dont il a traduit et commenté les œuvres complètes et à qui il a consacré de nombreux essais, André Pézard (1893-1984) a été l'un des acteurs les plus importants de l'italianisme français du XX siècle. Le volume André Pézard, autobiographe, italianiste, romaniste et médiéviste, paru aux Classiques Garnier en 2017, sous la direction de Michèle Gally et Elsa Marguin-Hamon, est l'aboutissement du colloque André Pézard en ses archives, organisé en 2014 pour présenter le fonds Pézard, qui venait d'être réuni aux Archives nationales ${ }^{1}$. Comme le précise Michèle Gally dans son essai introductif, " c'est bien des papiers de Pézard, portant les traces de son travail attentif, de ses semi-confidences, de ses corrections, que les intervenants se sont efforcés de comprendre l'œuvre achevée » (p. 24). Ainsi, l'exploration des archives du célèbre italianiste a été l'occasion, comme l'annonce le sous-titre du volume, d'esquisser le profil intellectuel de l'écrivain, de l'enseignant, du 
savant et du traducteur. Chacune de ces facettes se reflètent dans les quatre parties qui composent le volume: "À la genèse de l'œuvre ", "Un intellectuel et son temps ", « Dans l'atelier de l'érudit » et « L'art de traduire ».

Bien que le nom de Pézard soit étroitement lié à son œuvre savante, son premier livre est un récit autobiographique paru en 1918: Nous autres à Vauquois. L'auteur y relate, sous la forme d'un journal intime, son expérience d'un an et demi au front. Jean Norton Cru, dans Témoins (1929), analyse les témoignages des "poilus ", dont celui de Pézard. C'est à l'exemplaire de Nous autres à Vauquois annoté par Norton Cru (et conservé aujourd'hui à la bibliothèque universitaire d'Aix-en-Provence) qu'est consacré l'article de Marie-Françoise Attard-Maraninchi. Au-delà de l'intérêt spécifique pour l'analyse du récit de Pézard, l'étude d'Attard-Maraninchi est porteuse d'une réflexion sur la genèse des essais critiques, mobilisant l'étude des bibliothèques personnelles. La méthode de lecture et d'annotation de Cru y est présentée et mise en relation avec la genèse de Témoins. On remarquera la « vectorialité » des annotations déposées par Cru au fil des nombreuses campagnes de lecture : l'ouvrage (critique) en préparation guide sa lecture et le conduit à mettre en place un dispositif d'annotation très précis et très méthodique, comportant des repères et des aide-mémoires, mais aussi des commentaires à l'état embryonnaire. Ces procédés de marginalisation vont de pair avec la rédaction de notes, classées par catégories, organisées sous forme de rubriques titrées, et écrites sur le livre même, dans les pages liminaires: «loin de sacraliser l'ouvrage, Cru fait, de sa couverture, un support de travail » (p. 54). Par ce cas d'étude, Attard-Maraninchi retrace la genèse de Témoins, tout en explorant la mise au point progressive du jugement porté par le critique sur l'ouvrage de Pézard. Selon Norton Cru, qui fut lui-même soldat, le témoignage de Pézard est l'un des plus fidèles et des plus précis : une qualité qui repose, fort probablement, sur la genèse de Nous autres à Vauquois, étudiée par Philippe Lejeune. Pézard aurait en effet rédigé son récit autobiographique en s'appuyant sur cinq carnets où il avait noté, en prise directe, son expérience au quotidien pendant la guerre. À ces carnets, s'ajoutent les nombreuses lettres familiales, ainsi qu'un riche dossier iconographique, comportant environ cinq cents photos prises par le jeune soldat au front. Lejeune, après avoir classé ce dossier, se concentre sur les réécritures au cours desquelles la documentation privée (les cinq carnets, rédigés sous la forme d'un journal intime) est devenue récit publié. Pézard se serait tenu fidèlement à ses notes en ce qui concerne l'ordre chronologique et les données " événementielles » : l'agencement et la substance du récit ne font pas l'objet d'un remaniement de la part de l'auteur, qui pourtant ne publie pas ses carnets tels quels. Pézard travaille sur ses notes dans deux directions: d'une part, par la suppression de considérations répétitives ou peu significatives, de l'autre, par l'ajout de précisions et de détails émotifs, qui cependant ne falsifient jamais l'exactitude et la fiabilité du souvenir. C'est surtout sur le plan du style que Pézard intervient, dans un second temps, sur ses carnets, en vue de leur publication : comme l'illustre un exemple commenté par Lejeune, la comparaison entre les carnets, la version initiale du brouillon et le texte publié montre que les notes prises hâtivement par le jeune soldat ont fait l'objet d'un travail de réécriture à la recherche d'une plus grande élégance stylistique. Comme le précise Pézard dans une lettre destinée à Norton Cru, et publiée par Lejeune en annexe : « c'est une traduction plus qu'un remaniement » (p. 47).

3 La deuxième des quatre parties du volume porte sur le Pézard passeur de la culture et de la littérature italiennes en France. S'appuyant sur la transcription tapuscrite d'un 
entretien entre Pézard et Philippe Lejeune, Silvia Fabrizio-Costa retrace le parcours biographique et dégage les raisons qui ont poussé un jeune helléniste et germaniste prometteur à consacrer sa vie à l'italianisme, choix peu commun à l'époque. Il est évident que Pézard a été un interlocuteur important pour les spécialistes du Moyen Âge italien, même si sa fortune en Italie a été controversée : tout en étant un spécialiste reconnu et régulièrement cité, les études critiques de Pézard ont souvent été discutées et remises en question; en revanche, comme le montre l'article de Tobia Zanon, ses traductions ont rencontré un accueil très chaleureux de la part des Italiens.

Pour comprendre les quelques réserves portées aux interprétations de Pézard, il faut se pencher sur sa méthode, à laquelle est consacrée la troisième partie de cet ouvrage : face au problème philologique posé par la tradition de l'œuvre de Dante (il n'existe aucun autographe de Dante, d'où la nécessité de remonter par conjecture à un archétype, à travers la confrontation de ses multiples copies manuscrites) Pézard privilégie l'interprétation et, comme le montre Johannes Bartuschat, «semble vouloir ignorer le dilemme méthodologique [...] entre la fidélité aux manuscrits qui risquent de transmettre une lettre altérée ou incomplète, et la conjecture qui risque de remplacer le texte par ce que nous avons compris et qui nous empêche ainsi d'accéder à son sens " (p. 178).

5 Le problème de l'établissement du texte et celui de son interprétation sont bien évidemment préliminaires au travail de traduction: il faut choisir un texte et l'interpréter correctement pour pouvoir le transporter vers une autre langue. C'est bien au Pézard traducteur de Dante qu'est consacrée la quatrième partie de cet ouvrage. Pour rendre compte des difficultés auxquelles le traducteur de Dante est confronté, Francesca Manzari a proposé une confrontation entre les traductions pézardiennes de la Comédie publiée et celle de Jacqueline Risset, qui prend ses distances avec le projet de son prédécesseur : le cœur du problème est lié à la spécificité de la tradition italienne, dans la mesure où - à la différence du français - l'italien du Moyen Âge demeure assez proche de l'italien moderne, tout en gardant son étrangeté. Pézard s'efforce d'« offrir au lecteur français un texte à même de reconstituer la distance qui sépare Dante de son lecteur italien d'aujourd'hui » (p. 212) : pour ce faire, il « invente » une langue capable de garder la saveur de l'ancien français, par le recours à des archaïsmes lexicaux et syntaxiques, et par le choix de mots rares, désuets ou précieux. Comme le précise Jean-Charles Vegliante, qui s'appuie également sur son expérience de traducteur, «il n'est pas question là d'une langue nouvelle, mais bien plutôt d'écart linguistique : existant de fait pour les lecteurs italophones de Dante, mais à recréer pour le traducteur Pézard» (p.192). Cette quête linguistique est illustrée par les papiers autographes de Pézard, qui témoignent de son travail de traducteur : Vegliante, à l'aide de quelques exemples, esquisse un portrait du traducteur à sa table de travail. Les papiers préparatoires des traductions de Pézard montrent qu'il menait une longue réflexion en amont et que la première solution qu'il proposait se révélait souvent être la bonne : ses réécritures éclairent sa méthode, ainsi que son interprétation du texte source. Concernant la méthode, "tout se passe comme si les diverses possibilités avaient été testées d'emblée, lors de la première apparition du terme, puis monnayées [...] au long des occurrences suivantes " (p.197): les brouillons des traductions témoignent ainsi d'écarts minimes qui conduisent progressivement, par pas successifs, vers la forme définitive, très éloignée de la première proposition. Si l'on confronte les traductions des mêmes vers préparées par Pézard pour ses cours au Collège de France et celles rédigées, des années plus tard, pour les CEuvres complètes, on prend la mesure 
du trajet: les quelques exemples portés par Vegliante montrent le passage d'une traduction littérale, dont la visée est exclusivement herméneutique, à une traduction qui prend aussi en compte la métrique et la qualité de la langue, en un mot, la poésie de la langue de Dante.

Le volume se clôt sur un long essai, signé par Elsa Marguin-Hamon, qui présente le fonds Pézard aux Archives nationales, son histoire, son potentiel, et les critères qui en ont régi le classement. Par un survol passionnant de ces archives, Marguin-Hamon ouvre les précieuses pistes de recherche que les contributions de ce volume ont commencé à explorer : de la correspondance aux brouillons de Nous autres à Vauquois, des matériaux préparatoires pour les cours donnés au Collège de France aux brouillons des traductions, en passant par les journaux intimes, les dossiers iconographiques ou encore une partie de la bibliothèque personnelle de Pézard. D'un point de vue génétique, ces archives permettront d'explorer, par exemple, l'impact des spécificités et des genres des textes (autobiographie, essai critique, traduction, document privé) sur la méthode de travail d'un scripteur. De plus, par leur ampleur chronologique, elles se prêtent à l'étude de l'évolution du rapport à l'écriture : Quelles pratiques d'écriture se conservent, de l'adolescence à la maturité, et quelles autres se modifient au fil du temps? Sachant que Pézard a organisé personnellement, du moins en partie, ses papiers autographes, ses archives pourraient encore offrir un cas d'étude important pour une réflexion sur les "intentions d'archives", ce geste, autobiographique à sa manière, par lequel certains auteurs cherchent à livrer à la postérité une image de leur œuvre et donc d'eux-mêmes. Ce n'est là qu'une partie des nombreuses orientations de recherche qu'ouvrent les archives Pézard et qu'illustre la parution de ce volume.

\section{NOTES}

1. Après la mort de Pézard, une partie de ses archives a été déposée par le Collège de France à l'IMEC, en 2001, et a fait l'objet d'un premier classement, avant d'être transférée, en 2012, aux Archives nationales, à qui la famille de Pézard (Sylvie Pézard, Daniel Roche et Olivier Roche) avait également donné, en 2011, une grande partie des papiers qui étaient restés chez sa fille Sylvie. En 2013, une fois le fonds Pézard réuni, Elsa Marguin-Hamon a commencé le classement des papiers (fonds Pézard, 691AP), ce qui en a permis l'exploitation. 


\section{AUTEURS}

\section{MONICA ZANARDO}

Monica Zanardo est membre de l'équipe Écritures des Lumières de l'ITEM, où elle participe aux activités du groupe de travail Manuscrits italiens. Ses recherches portent sur les archives littéraires italiennes et sur les bibliothèques d'écrivains (XVIII ${ }^{\mathrm{e}}-\mathrm{XX}^{\mathrm{e}}$ siècles). Elle est auteure de l'essai Il poeta e la grazia. Una lettura dei manoscritti della Storia di Elsa Morante (Rome, Edizioni di Storia e Letteratura, 2017) consacré au dossier de genèse du roman La Storia d'Elsa Morante ; à présent, elle prépare l'édition savante numérique des archives littéraires de Vittorio Alfieri.

monicazanardo@gmail.com 